\title{
Histiocitoma fibroso maligno retroperitoneal con infiltración de órganos vecinos
}

\author{
Gimeno Argente V, Bosquet Sanz M, Gómez Pérez L, Delgado Oliva FJ, Arlandis Guzmán S, \\ Jiménez Cruz JF.
}

Servicio de Urología. Hospital Universitario La Fe. Valencia.

Actas Urol Esp. 2007;31(5):562-566

\section{RESUMEN \\ HISTIOCITOMA FIBROSO MALIGNO RETROPERITONEAL CON INFILTRACIÓN DE ÓRGANOS VECINOS}

Los tumores retroperitoneales primarios son neoformaciones extremadamente raras, de naturaleza maligna en la mayoría de los casos. Presentamos el caso de un varón de 48 años con una gran masa retroperitoneal detectada durante el estudio de un síndrome constitucional. La masa fue tratada quirúrgicamente y el diagnóstico anatomopatológico fue de histiocitoma fibroso maligno. Realizamos una revisión de la literatura y analizamos su presentación clínica, hallazgos histológicos, pruebas diagnósticas de imagen y manejo terapéutico.

Palabras clave: Retroperitoneo. Tumor retroperitoneal. Histiocitoma fibroso maligno.

\section{ABSTRACT}

\section{RETROPERITONEAL MALIGNANT FIBROUS HISTIOCYTOMA WITH CONTIGUOUS ORGANS INFILTRATION}

Retroperitoneal tumours are extremely rare neoplasms, most of them malignant. We described the case of a 48-year-old man with a large retroperitoneal mass detected during the study of a constitutional syndrome. The mass was treated surgerically and pathological diagnosis was malignant fibrous histiocytoma. Literature is reviewed and clinical features, histological findings, radiological techniques and therapeutic management are analyzed.

Keywords: Retroperioneum. Retroperitoneal tumour. Malignant fibrous histiocytoma.

$\mathrm{E}^{1}$ retroperitoneo es un espacio real que contiene estructuras de origen meso y ectodérmico, así como sus remanentes embrionarios. Los tumores retroperitoneales primarios son neoformaciones benignas o malignas procedentes de este espacio, excluyendo aquellas derivadas de los órganos y grandes vasos situados en él. Su frecuencia oscila entre el 0,2 y el 0,6 \% del total de tumores y en la mayoría de ocasiones son de naturaleza maligna (entorno a un $85 \%$ de casos), correspondiendo un $35 \%$ de ellos a sarcomas, entre los que se encuentra el histiocitoma fibroso maligno $(\mathrm{HFM})^{1,2}$.
Presentamos un caso de HFM retroperitoneal de grandes dimensiones, con infiltración masiva de órganos adyacentes y pronóstico sombrio a pesar de tratamiento quirúrgico.

\section{CASO CLÍnICO}

Varón de 48 años de edad, sin antecedentes médicos de interés salvo un traumatismo craneoencefálico 13 años antes, intervenido de apendicectomía, discectomía L5-S1 y circuncisión, que es remitido a consulta de Urología por dolor en flanco derecho, anemia ferropénica y síndrome constitucional de seis meses de evolución. A la 
exploración física presenta buen estado general, sin detectarse masas tras la palpación abdominal. Los datos analíticos sanguíneos son normales salvo hemoglobina de $10.5 \mathrm{~g} / \mathrm{dl}$, hematocrito de $32.4 \%$ y VSG a la primera hora de $52 \mathrm{~mm}$. Los marcadores tumorales sanguíneos CEA, Ca 19,9 y alfafetoproteina, son normales. Ante la sospecha de proceso neoformativo, se practica TC abdomino-pélvico con contraste intravenoso,

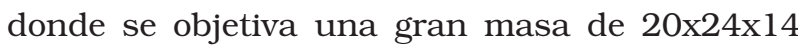
$\mathrm{cm}$, que ocupa el hemiabdomen derecho hasta la cresta ilíaca, con desplazamiento del hígado, vesícula biliar y páncreas, así como de los vasos retroperitoneales, de estructura heterogénea con áreas hipodensas, calcificación central de tipo radial y captación irregular del contraste, sugestivo de origen renal (Figs. 1a y 1b).
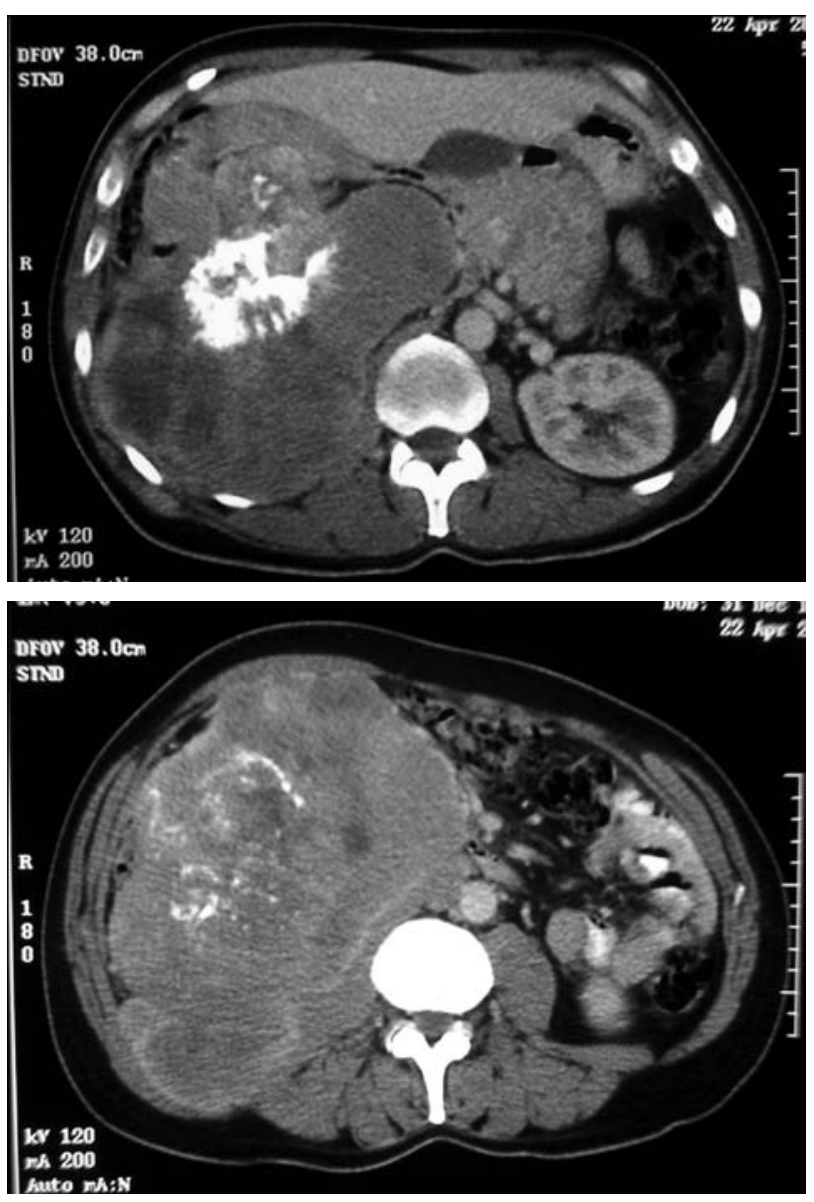

FIGURAS 1a y 1b: Imágenes de TC abdomino-pélvico con contraste intravenoso, que muestra una gran masa de 20 x $24 \times 14 \mathrm{~cm}$. que ocupa el hemiabdomen derecho hasta la cresta iliaca, con desplazamiento de órganos y vasos retroperitoneales, de estructura heterogénea con áreas hipodensas, calcificación central de tipo radial y captación irregular del contraste.
Se procede a intervención quirúrgica con hallazgos intraoperatorios de masa retroperitoneal que infiltra riñón derecho, colon ascendente y cabeza de páncreas (Fig. 2), por lo que se practica la exéresis del tumor retroperitoneal, asociando nefrectomía y hemicolectomía derecha, así como pancreatectomía cefálica.

El estudio histológico describe una masa tumoral de 4300 gramos (Fig. 3), de superficie abollonada y consistencia dura, que invade la cortical renal y la grasa del hilio renal, al igual que uréter, pared de colon y tejido pancreático. Microscópicamente corresponde a una proliferación tumoral maligna de estirpe mesenquimal, que crece con un patrón predominantemente estoriforme, constituida por haces entrelazados en todas direcciones, de elementos celulares fusiformes y de numerosas células multinucleadas gigantes pleomórficas, todo ello sustentado por una densa trama fibrilar de colágeno y reticulina (Fig. 4). En el seno del tumor hay frecuentes áreas metaplásicas de calcificación y osificación, así como algunos focos de necrosis tumoral. El perfil inmunohistoquímico de las células tumorales muestra positividad para CD68 y Ki67, y negatividad para desmina y actina. Estos hallazgos son compatibles con el diagnóstico anatomopatológico de histiocitoma fibroso maligno pleomórfico-estoriforme.

Tras la intervención, el paciente permanece durante 24 horas en el Servicio de Reanimación con buena evolución en el postoperatorio inmediato. Durante el sexto día de ingreso hospitalario, presenta un cuadro de disnea intensa, sudoración profusa y palidez cutánea, con temperatura axilar de $38,2{ }^{\circ} \mathrm{C}$, presión arterial de 117/68 $\mathrm{mmHg}$, frecuencia cardiaca de 125 lat./min y saturación de oxígeno del $75 \%$. La auscultación pulmonar muestra hipoventilación generalizada y en el análisis sanguíneo destaca leucocitosis de 22600 con neutrofilia del 91\%. Estos hallazgos motivan su ingreso en la Unidad de Cuidados Intensivos donde se decide intubación orotraqueal y ventilación mecánica, ante el importante trabajo respiratorio y la progresiva desaturación de oxígeno. A los pocos minutos de la intubación presenta bradicardia progresiva que se sigue de disociación electromecánica, por lo que se inician maniobras de reanimación cardiopulmonar avan- 


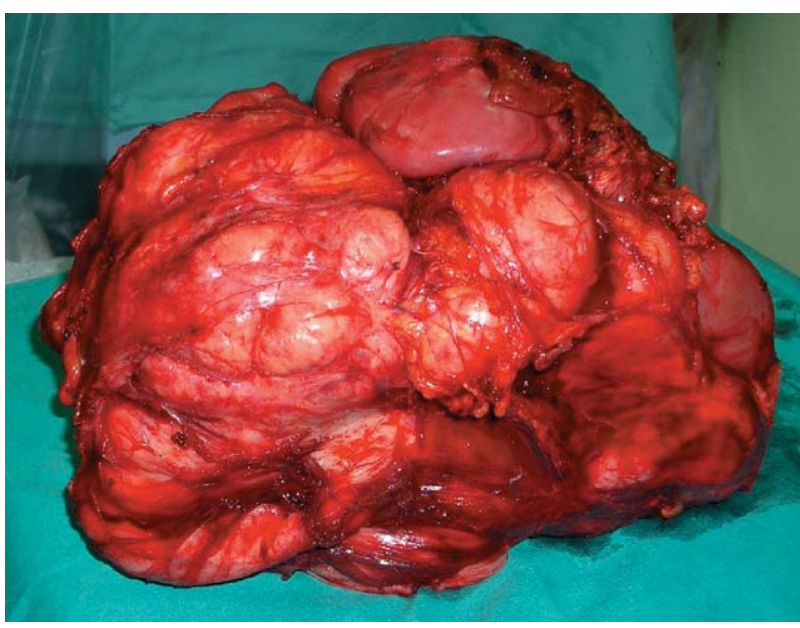

FIGURA 2: Tumor retroperitoneal de grandes dimensiones, que infiltra riñón derecho, colon ascendente y cabeza de páncreas.

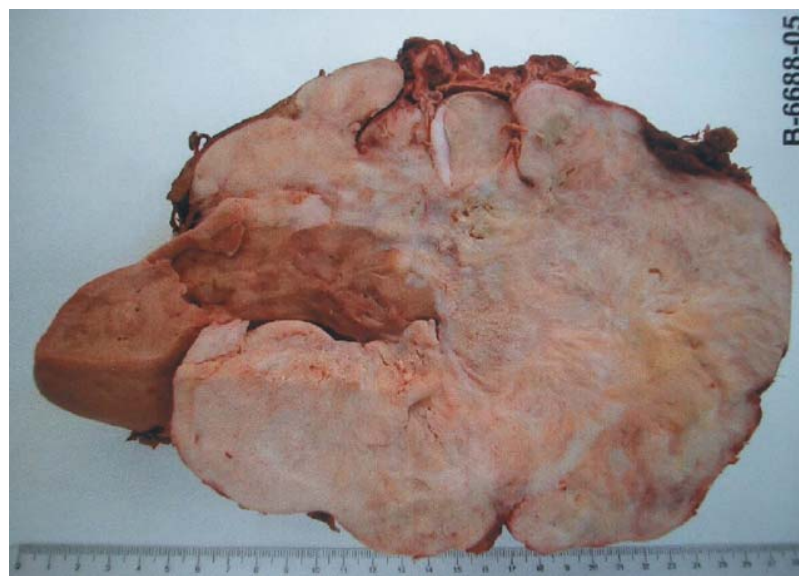

FIGURA 3: Sección macroscópica de masa tumoral ama rillenta de dimensiones $25 \times 18 \times 15$ cms. y peso 4300 grs., de distribución lobulada con áreas calcificadas $u$ osificadas y que engloba parcialmente en su interior riñón, uréter, pared de colon y tejido pancreático.

zada. Durante las mismas, ocurren varios episodios de fibrilación ventricular que precisan de choques eléctricos, seguidos de una asistolia de la que no se recupera. El paciente fallece a consecuencia de insuficiencia respiratoria aguda secundaria a neumonía nosocomial.

\section{DISCUSIÓN}

El histiocitoma fibroso maligno es el sarcoma de tejidos blandos más común del adulto ${ }^{3,4}$. La mayor incidencia se sitúa entre la $5^{\mathrm{a}}$ y $7^{\mathrm{a}}$ década de la vida, siendo inusual su presentación antes de los 40 años $^{5}$. Presenta cierta predilección por el sexo masculino y se localiza predominante-

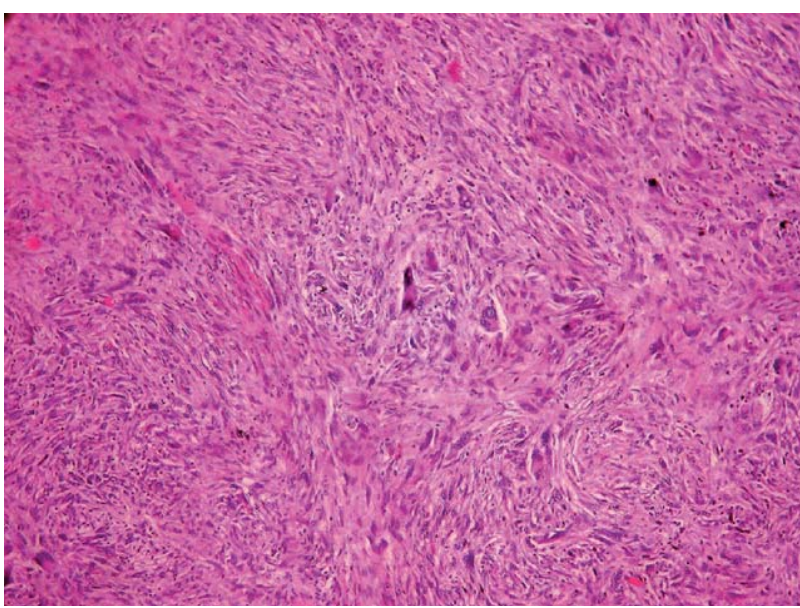

FIGURA 4: Imagen microscópica de anatomía patológica que muestra una proliferación tumoral maligna de estirpe mesenquimal, formada por haces entrelazados de células fusiformes y multinucleadas gigantes pleomórficas, sustentadas por una trama fibrilar de colágeno, con patrón de crecimiento estoriforme.

mente en extremidades, siendo el retroperitoneo el segundo lugar en frecuencia con tan sólo un $12-15 \%$ de $\operatorname{casos}^{6-8}$.

La localización retroperitoneal de los tumores permite el desarrollo de los mismos sin apenas generar manifestaciones clínicas. De ahí que estos tumores alcancen un gran tamaño antes de provocar síntomas por compresión o infiltración de órganos vecinos, como en el caso que nos ocupa, lo que explicaría el pobre pronóstico de estos pacientes ${ }^{9}$.

El origen de este tumor continúa siendo desconocido hoy día. La hipótesis histogenética más aceptada en la actualidad es la que sitúa a las células mesenquimales pluripotenciales como precursoras neoplásicas, lo que justifica que en ocasiones coincida temporalmente con otros tumores como el liposarcoma o el fibrosarco$\mathrm{ma}^{1,4,10}$.

Macroscópicamente, estos tumores adquieren un gran tamaño, son multinodulares y pobremente encapsulados, con áreas de hemorragia y necrosis, como otros sarcomas de alto grado $^{11}$.

Histológicamente estas neoplasias se caracterizan por su amplio pleomorfismo y por la presencia de histiocitos y células fibroblásticas. Se han definido diversas variantes histológicas: fibroso de células gigantes, inflamatorio y mixoide. Entre los patrones de crecimiento tumoral distinguimos el 
estoriforme o "en rueda de carro", fascicular y pleomórfico, siendo este último el más frecuen$\mathrm{te}^{5}$. En el caso que presentamos, encontramos un crecimiento mixto pleomorfico-estoriforme constituido por células multinucleadas gigantes pleomórficas en forma de huso o estrella, formando haces entrelazados en todas direcciones, todo ello sustentado por una matriz mixoide rica en mucopolisacáridos.

El perfil inmunohistoquímico de las células tumorales suelen mostrar positividad para los marcadores CD68, quimiotripsina, tripsina $\mathrm{y}$ Ki67 ${ }^{1}$.

Clínicamente, el sintoma inicial suele ser el dolor del flanco afecto, aunque a veces puede presentarse como síndrome constitucional con astenia, anorexia y adelgazamiento o síndrome febril $^{10}$. Otros signos son los derivados del incremento de la presión abdominal, como distensión abdominal, varicocele y/o hernia de la pared abdominal $^{6,12}$. Ocasionalmente podemos detectar masa abdominal a la palpación, al igual que anemia, leucocitosis, elevación de la VSG y/o productos ureicos nitrogenados, en los análisis sanguíneos.

Entre las pruebas diagnósticas de imagen, la radiografia simple y la urografia intravenosa proporcionan datos inespecíficos, como borramiento de la línea del psoas, calcificaciones focales o difusas o desplazamiento de estructuras retroperitoneales. La ecografía y en especial la TC resultan especialmente útiles al aportar información acerca de la composición y densidad, extensión y relación con órganos y estructuras adyacentes ${ }^{13}$. Ecográficamente estos tumores suelen presentar un patrón hipoecogénico con algunos ecos internos, aunque en ocasiones muestran un patrón mixto $^{1}$. El TC usualmente evidencia neoformaciones de aspecto sólido, de densidad heterogénea con captación de contraste, presentando zonas de hipodensidad en su interior que corresponden a áreas de necrosis y hemorragia ${ }^{11}$. Esta prueba proporciona información sobre la naturaleza tumoral (importante en el diagnóstico diferencial de neoplasias malignas en esta localización) y ayuda a delimitar el tamaño y localización del tumor, lo que facilita el abordaje quirúrgico y el seguimiento postoperatorio. Algunos autores destacan la importancia de la resonancia magnética en el estudio de estas neoplasias, ya que delimita las relaciones anatómicas y puede definir la malignidad con bastante precisión ${ }^{12}$.

$\mathrm{El}$ único tratamiento con posibilidades curativas es el quirúrgico y se basa en la resección completa del tumor ${ }^{1,4,5,7,14}$. Esto implica que en ocasiones sea necesario la extirpación de órganos y estructuras adyacentes que han sido infiltradas por el tumor, de ahí el pronóstico tan ensombrecedor que puede alcanzar este tumor, justamente como en el paciente de nuestro caso.

El comportamiento agresivo de estos tumores justifica una supervivencia a los dos años alrededor del $60 \%$, con un alto porcentaje de recidiva local y con tendencia a metastatizar en pulmón (órgano más frecuentemente afectado), hígado, hueso y médula ósea ${ }^{1,5}$.

Otros tratamientos alternativos a la cirugía, como la radioterapia y la quimioterapia, continúan siendo objeto de controversia aunque no parecen aumentar la supervivencia local ${ }^{15}$. La radioterapia local postoperatoria a altas dosis podría resultar útil en el control local de la enfermedad, aunque todavía no está claro el beneficio que aporta $^{5}$. De igual forma, se ha utilizado distintos quimioterápicos en régimen aislado o en combinación, sin obtener resultados satisfactorios con ninguno de ellos. Actualmente se cree que la única combinación efectiva es la que incluye Adriamicina, DTYC (dimetil-triazeno-imidazolcarbosamida) y Vincristina ${ }^{12}$. Por tanto, la cirugia puede considerarse la única opción válida con intención curativa y otros tratamientos sólo son adyuvantes o paliativos ${ }^{16}$.

\section{REFERENCIAS}

1. Argüelles Salido E, Congregado Ruiz CB, Medina López RA, Pascual del Pobil Moreno JL. Histiocitoma maligno fibroso retroperitoneal. Actas Urol Esp. 2004;28(8):624-626.

2. García Freire C, Villar Núñez M, López Pita J, Martínez Cruceiro S. Histiocitoma fibroso benigno retroperitoneal. Presentación de un caso. Actas Urol Esp. 1998;22(6):524-527.

3. Fignon A, Marret H, Body G, Saile N, Lanson Y, Le Floch O et al. Retroperitoneal malignant fibrous histiocytoma. Ann Chir. 1997;51(9):1023-1027.

4. Zuluaga Gómez A, Torres Ramírez C, García Gil JM, Gómez Morales M, Espejo Maldonado E, Puebla Ceverino M. Tumores retroperitoneales primitivos de origen histiocitario. Actas Urol Esp. 1984;8(4):279-288.

5. Giménez Bachs JM, Salinas Sánchez A, Lorenzo Romero J, Donate Moreno MJ, Hernández Millan IR, Segura Martín M et al. Fibrohistiocitoma maligno retroperitoneal de gran tamaño: aportación de un caso. Arch Esp Urol. 2004;57(2): 172-174. 
6. Virgili G, Di Stasi SM, Storti L, Orlandi A, Vespasiani G. Successful management of retroperitoneal malignant fibrous histiocytoma involving both kidneys. Scand J Urol Nephrol. 2000;34(3):208-210.

7. Weiss SW, Enzinger FM. Malignant fibrous histiocytoma: an analysis of 200 cases. Cancer. 1978;41(6):2250-2266.

8. Kearney MM, Soule EH, Ivins JC. Malignant fibrous histiocytoma: a retrospective study of 167 cases. Cancer. 1980; 45(1): 167-178.

9. Povar J, Franco JM, Muñoz JR, Horndler C, Arazo P, Portoles A, et al. Malignant fibrous histiocytoma of soft tissue: description of 10 cases. Med Clin (Barc). 1991;96(1): 6-10.

10. Garmendia Larrea JC, Arocena Lanz F, Sanz Jaca JP, Lopez García JA, Ruiz Diaz I, Sanroma Ortueta I. Histiocitoma fibroso maligno retroperitoneal tipo inflamatorio. A propósito de un caso. Arch Esp Urol. 1989;42(9): 922925.

11. Goldman SM, Hartman DS, Weiss SW. The varied radiographic manifestations of retroperitoneal malignant fibrous histiocytoma revealed Through 27 cases. J Urol. 1986; 135(1):33-38.

12. Herrero Payo JA, Paez Borda A, Aramburu J, Acebal Lucia J, Sánchez Sánchez E, Llorente Abarca C, et al. Histio- citoma fibroso maligno retroperitoneal recidivante tipo mixoide con infiltración de mesocolon. Arch Esp Urol. 1994;47(7):722-725.

13. Repassy D, Csata S, Sterlik G, Hazslinszhy P. Retroperitoneal malignant fibrous histiocytoma. Int Urol Nephrol. 1999;31(3):303-311.

14. Gomez de la Torre R, Triviño A, Pozuelo Manchon M, Rubio Barbon S. Histiocitoma maligno del retroperitoneo: un diagnóstico complicado. An Med Interna. 1994;11(6):307308.

15. Shiloni E, Szold A, White DE, Freund HR. High-grade retroperitoneal sarcomas role of an aggressive palliative approach. J Surg Onc. 1993;53(3):197-203.

16. Eilber FC, Eilber KS, Eilber FR. Retroperitoneal sarcomas. Curr Treat Options Oncol. 2000;1(3):274-278.

Correspondencia autor: Dr. V. Gimeno Argente Servicio de Urología. Hospital Universitario La Fe. Avda. Campanar, 21 - 46009 Valencia. Tel.: 963862700 E-mail autor: vigiar71@hotmail.com Información artículo: Nota clínica

Trabajo recibido: junio 2006

Trabajo aceptado: agosto 2006 